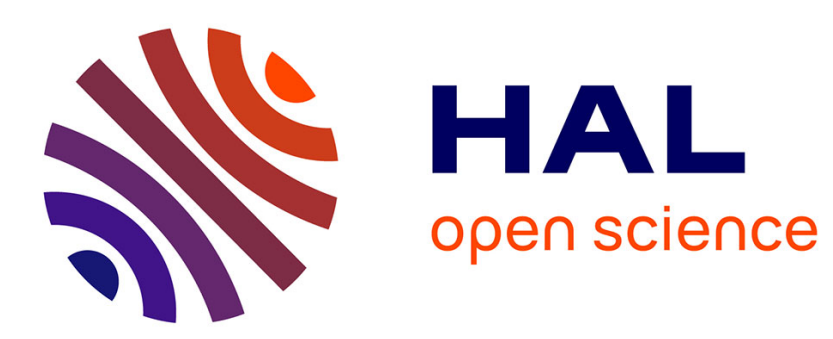

\title{
A heliospheric hybrid model: hydrodynamic plasma flow and kinetic cosmic ray transport
}

K. Scherer, S. E. S. Ferreira

\section{To cite this version:}

K. Scherer, S. E. S. Ferreira. A heliospheric hybrid model: hydrodynamic plasma flow and kinetic cosmic ray transport. Astrophysics and Space Sciences Transactions, 2005, 1 (1), pp.17-27. hal00297470

\section{HAL Id: hal-00297470 \\ https://hal.science/hal-00297470}

Submitted on 3 Mar 2005

HAL is a multi-disciplinary open access archive for the deposit and dissemination of scientific research documents, whether they are published or not. The documents may come from teaching and research institutions in France or abroad, or from public or private research centers.
L'archive ouverte pluridisciplinaire HAL, est destinée au dépôt et à la diffusion de documents scientifiques de niveau recherche, publiés ou non, émanant des établissements d'enseignement et de recherche français ou étrangers, des laboratoires publics ou privés. 


\title{
A heliospheric hybrid model: hydrodynamic plasma flow and kinetic cosmic ray transport
}

\author{
K. Scherer ${ }^{1}$ and S. E. S. Ferreira ${ }^{2}$ \\ ${ }^{1}$ Institut für Astrophysik und Extraterrestrische Forschung der Universität Bonn, Auf dem Hügel 71, D-53121 Bonn, Germany \\ ${ }^{2}$ Unit for Space Physics, School of Physics, North-West University, 2520 Potchefstroom, South Africa
}

Received: 15. December 2004 - Revised: 11. February 2004 - Accepted: 15. February 2004 - Published: 3 March 2005

\begin{abstract}
In this paper we present a new five particle species hybrid model for calculating cosmic ray particle transport and acceleration in a dynamic heliospheric environment. In particular the effects of solar cycle related changes in the solar wind speed on the heliospheric geometry, solar wind flow and cosmic ray distribution are discussed, when a polarecliptic asymmetry at the inner boundary is modeled. It is shown that the disappearance of the fast solar wind over the solar poles toward solar maximum influences the geometry of the termination shock which is an important structure for cosmic ray acceleration. For solar maximum conditions, the shock radius is smaller in the polar regions and in the heliospheric tail compared to solar minimum. These changes influence cosmic ray transport and acceleration in these regions, especially for the polarity cycle where positive particles drift in along the heliospheric current sheet. For this polarity cycle, and for both the anomalous and galactic cosmic ray protons, an increase in particle intensities at the shock in the heliospheric tail is computed as the shock moves inward toward the Sun. For the heliospheric nose, it is also shown that both the plasma speed and cosmic ray intensities are relative insensitive to changes in the latitudinal profile of the solar wind speed. Therefore toward solar maximum conditions there is a decrease in the nose-tail asymmetry of the computed cosmic ray distribution compared to solar minimum conditions.
\end{abstract}

\section{Introduction}

The theoretical discussion of the interaction between the solar wind and the local interstellar medium (LISM) has a long standing history, starting from early publications by Parker (1963) and by Baranov et al. (1971). Depending on the question whether or not the inflow of interstellar plasma is subor supersonic, the interaction scenario produces one or two

Correspondence to: K. Scherer

(kscherer@astro.uni-bonn.de) shock fronts, the inner, the termination shock of the solar wind, and the outer, the bowshock of the interstellar ionized matter. The interstellar wind is a consequence of the relative motion between the solar system and the local interstellar medium, while the inner solar wind results from the solar coronal outflow with asymptotically supersonic velocities. In the interface region between the two shocks a contact discontinuity forms, the heliopause, which separates both media by a surface which cannot penetrated by the plasmas.

It became clear quite early, that the interaction of the plasma flows with the incoming interstellar neutral atoms, especially hydrogen (e.g. Fahr, 1968; Blum and Fahr, 1970; Holzer, 1977) plays an essential role in the physics of the outer heliosphere. Especially the pickup ions, which are created as consequence of charge exchange processes of $\mathrm{H}$ atoms with solar wind ions (e.g. Fahr, 1983; Isenberg et al., 1985; Fahr et al., 1985; Ruciński and Fahr, 1991; Zank and Pauls, 1997; Fahr and Ruciński, 1999) came in the focus of interest, because they are considered to be the seed population for anomalous cosmic rays (ACR). The latter was discovered in the early 1970 (by Garcia-Munoz et al., 1973) as an additional component to the galactic cosmic rays (GCRs) (for more details see below).

Up to now the only model which describes a dynamical coupling of all these five fluids (protons, hydrogen, pickup ions, ACRs and GCRs) in a self-consistent manner is the so-called Bonn-model (Fahr et al., 2000), which, as was recently demonstrated, also allows to include time variable solar wind ram pressure conditions, e.g. periodic changes in the plasma parameters caused by the solar activity cycle (Scherer and Fahr, 2003a,b; Scherer et al., 2004). Without the high energy particles but with protons and hydrogen, the dynamics of the heliosphere is also modeled by Zank and Müller (2003), while earlier dynamic models included only protons (Steinolfson, 1994; Liewer et al., 1995; Karmesin et al., 1995; Whang and Burlaga, 1993; Baranov and Zaitsev, 1998; Tanaka and Washimi, 1999). Recently, Izmodenov and Malama (2004) have also presented a mono-periodic timedependent model including $\mathrm{H}$-atoms which were described 
by a Monte-Carlo method, however, no high energy plasma components were included. Recently, high energy particles are also included in a static model by Alexashov et al. (2004).

In more recent models the heliosphere was modeled in three dimensions (e.g. Washimi and Tanaka, 1996; Zank and Pauls, 1996; Borrmann and Fichtner, 2005), some of them even incorporated the heliospheric magnetic field (Ratkiewicz et al., 1996, 1998; Linde et al., 1998; Pogorelov and Matsuda, 1998, 2000; Pogorelov et al., 2004). While most of the above cited models use a hydrodynamical approach to describe H-atoms, a few have used more complicated approaches like Boltzmann-kinetic descriptions (Ripken and Fahr, 1983; Fahr, 1991, 1996; Osterbart and Fahr, 1992; Baranov and Malama, 1993; Müller et al., 2000; Izmodenov, 2001). Recent reviews of the hydrodynamical heliosphere modeling can be found in Zank (1999) and Fahr (2004).

The heliosphere resulting under the relative motion between the LISM and the Sun has an asymmetric structure, for instance yielding a ratio of the upwind-to-downwind termination shock distance of approximately 1:2. In addition, the inner heliosphere is also more elongated in the poleward directions because of the latitudinal variation of the solar wind momentum flux, a fact which was revealed by observations with the Ulysses spacecraft (Phillips et al., 1995; McComas et al., 2000). This quantity increases by a factor of $\sim 1.5$ from the equatorial regions toward the poles, suggesting a pole-ward elongated heliospheric termination shock. Nevertheless, the mass flux is reported to be fairly constant with latitude and over a solar cycle McComas et al. (2003), while the dynamic pressure is changing, leading to a more pronounced asymmetry during solar minimum conditions.

The basics for the description of the heliospheric cosmic ray distribution was again presented by Parker (1965) deriving the transport equation for high energetic particles in the heliosphere. The development of these theories in the recent years is nicely described by Fichtner (2001, 2005). While the galactic cosmic rays (GCRs) originate outside the solar system (e.g. Axford et al., 1978; Bell, 1987; Blandford and Ostriker, 1978), the anomalous cosmic rays (Fisk et al., 1974; Pesses et al., 1981; Cummings et al., 2002) are accelerated inside the heliosphere (for a review see Fichtner, 2001). A lot of effort was spent in the description of the diffusion tensor (Jokipii, 1966; Bieber et al., 1994; Burger and Hattingh, 1998; Giacalone and Jokipii, 1999; le Roux et al., 1999; Lerche and Schlickeiser, 2001; Ferreira et al., 2001; Florinski et al., 2003), or in the modeling of asymmetries in the heliospheric structure (Donohue and Zank, 1993; Chalov and Fahr, 1994; Kòta and Jokipii, 1998; Krimigis et al., 2003; McDonald et al., 2003). Only recently the initial spectra of the GCRs in the LISM have been studied in detail by Moskalenko et al. (2002) and Langner (2004). The transport equation was solved numerically with different assumptions (see Potgieter, 1998, for a detailed discussion), for example 3D steady state models (e.g. Hattingh and Burger, 1995; Zhang, 1999a,b) or fully 3D-time dependent models (Kòta and Jokipii, 1998; Kissmann et al., 2003), among others. In the Potchefstroom model (Potgieter, 1993, 1995, 1998; Ferreira et al., 2001; Ferreira and Potgieter, 2004) the complete transport equation is solved and applied to the modulation of galactic electrons, protons, as well as heavier ions.

In almost all transport models described above a relative simple heliospheric structure was used, e.g. a spherical heliosphere and a radial decaying solar wind between the termination shock and the heliopause. The effect of a poleward elongated and realistic heliospheric geometry on cosmic ray transport were studied in previous work (Ferreira et al., 2004; Ferreira and Scherer, 2004), in which the structure of the heliosphere as well as the plasma parameters were taken from the Bonn model without the GCR and ACR components.

In the present work, the two approaches, the hydrodynamic plasma code (the Bonn model) and the kinetic solution of the transport equation for cosmic rays (Potchefstroom model) have been combined into a self consistent five species hybrid code. This hybrid model also allows to study the effects of changes related to the solar activity cycle. Additionally, the changes with the solar wind latitudinal profile and its consequences on the heliospheric geometry and cosmic ray transport have been implemented into the hybrid code. Concerning the cosmic ray transport we report on the modulation of anomalous (ACR) and galactic (GCR) proton cosmic ray intensities in the heliosphere, and show how these are affected by dynamic changes in the solar wind speed.

\section{The Hydrodynamic Model}

\subsection{The stationary model}

The theoretical basics of the 5-species Bonn fluid model have already been published by Kausch (1998), Fahr et al. (2000), and Fahr (2000). Thus, we give here only a short overview. The Bonn model includes consistently, besides protons of solar and interstellar origin, also interstellar H-atoms, heliospheric H-pick-up ions, proton ACR's and GCR's as separate fluids. These five fluids are dynamically and thermodynamically coupled to build the multifluid interaction system. The protons and the hydrogen are coupled by charge exchange processes, which in turn produce a new component, the pickup Ions (PUIs) which are comoving with the local solar wind, but thermodynamically behave as an independent fluid with separate values of density, temperature, sound speed and pressure. The PUI seed population is injected at the shock, with a rate which depends on its local strength, into the Fermi-I acceleration process to create the high energetic anomalous cosmic ray (ACR) component (for details see Fahr et al., 2000). The injection rate corresponds to a specific fraction of the PUI flux which locally passes over the shock. This injection constitutes a local ACR source at the shock which is taken into account in an energyaveraged ACR transport equation describing convection and spatial diffusion of this high-energy $10 \mathrm{MeV} /$ nuc fluid. Finally, the GCR's have been included by coupling their partial pressure to the total pressure using an energy-averaged GCR 
transport equation. Hereby the coupling occurs due to convective GCR changes connected with the local plasma bulk flow, and due to a modulation of the plasma motion by ACR and GCR pressure gradients. The energy density, i.e. the dynamic pressure, of the cosmic rays contributes to the dynamic structure of the heliosphere.

However, in this new hybrid approach the ACR and GCR intensities, in the form of spectra, are computed by solving the Parker (1965) transport equation which also takes into account energy changes that these particles may experience (Potgieter, 1998; Ferreira et al., 2004; Ferreira and Scherer, 2004). To take care of the fact that in our new approach, the pressures of the high energetic particles, e.g. GCRs and ACRs, are important, they are calculated by integrating the corresponding energy spectra. In detail: First, the proton, hydrogen and PUI species are calculated hydrodynamically, secondly, the corresponding GCR and ACR spectra are estimated with the kinetic model described below. In the third step, these spectra are integrated to get the dynamic pressure of the high energy particles, which then is added to the total pressure of the five fluid system. Then with the new total pressure terms the evolution of hydrodynamic fluids is calculated. This scheme is applied, until the a stationary state is reached. This stationary state is then used as initial condition for the dynamic model, in which the inner boundary conditions change periodically, as described in the next section.

A coupling between the plasma and the ACR and GCR component is also included via the solar wind speed and the heliospheric magnetic field (see below). Moreover, the divergence of the proton speed is responsible for the acceleration of the ACRs which, in turn, react back to the heliospheric structure by their pressure. Moreover, a fraction of the PUIs are used as seed of the ACRs, which therefore, depend on the hydrogen density of the LISM and the coupling to the solar wind protons. A detailed analysis of the ACR acceleration and coupling to the heliospheric structure will be given in a forthcoming paper.

\subsection{The dynamic model}

To describe the GCR and ACR spectra at different time phases of the solar activity cycle, one primarily needs a detailed knowledge of the time-dependent properties of the background plasma flows, i.e. the solar wind and the interstellar plasma flow. To obtain the GCR and ACR flux intensities, they have to be coupled consistently to the other differential equations describing dynamics and thermodynamics of the low-energy plasma constituents. In the Bonn model this was realized using energy averaged equations for the high energy species. In the new model the approach is changed, but the principles of the dynamic modeling are the same. Therefore, a description of the dynamic modeling follows based on the observations in the outer heliosphere.

Variations of the solar wind pressure connected with the solar activity cycle have been reported on the basis of deep space probe data obtained with PIONEER and VOYAGER spacecraft (Kayser et al., 1984; Lazarus and McNutt, 1990) and they essentially show cycle-periodic variations of the dynamical pressure roughly by a factor 2 with maxima delayed up to 4 years with respect to the activity cycle maximum. As can be extracted from data presented by Lazarus and Belcher (1987), Gazis (1994) or McComas et al. (2000) the solarcyclic variation of the solar wind dynamical pressure is connected with a nearly constant solar wind mass flux, since solar wind velocity and density appear to be appropriately anticorrelated (McComas et al., 2004). This time-dependence and the its reaction to the whole interface system due to the dynamically changing solar wind pressure is described within the fully-time-dependent HD-simulation of the five dynamically relevant fluids, in which the solar wind mass flow is constant during the solar cycle.

\subsubsection{The dynamics of the solar wind}

Hereby we follow VOAYGER-1/2 data taken by Gazis (1994) and try to best-fit them by the following analytic timedependencies: For the solar wind bulk flow velocity $V_{s}$ we adopt (for details see Scherer and Fichtner, 2004):

$$
\begin{aligned}
& f(t)=a_{s}+b_{s} \cos \left(\omega_{s} t\right) \exp \left[S \cos \left(\omega_{s} t\right)\right] \\
& V_{s}(t)=V_{\min }+\Delta V_{s} f(t)
\end{aligned}
$$

with the solar cycle period $\tau_{s}=2 \pi / \omega_{s}=11 \mathrm{yrs}$, and with $V_{\min }=300 \mathrm{~km} / \mathrm{s}$ and $\Delta V_{s}=v_{\max }-v_{\min }=500 \mathrm{~km} / \mathrm{s}$. The constants $a_{s}, b_{s}$ are chosen to normalize the function $f$ to values between 0 and 1 , e.g. $a_{s}=b_{s} e^{-S}$ and $b_{s}=1 /\left(e^{S}+e^{-S}\right)$, while $S$ controls the steepness of the function and the width of the extrema. As discussed in Scherer and Fahr (2003a) and Scherer and Fichtner (2004) the solar wind mass flow $\Phi_{m s}$ is constant in time (see also McComas et al., 2004) and hence the proton density anticorrelates with the bulk velocity like:

$n_{s}(t)=\frac{\Phi_{m s}}{V_{\min }+\Delta V_{s} f(t)}$

Therefore, the most relevant dynamical quantity, the dynamical pressure $\Pi_{s}$ at the inner boundary of our simulation program is given by:

$\Pi_{s}(t)=\Phi_{m s}\left[V_{\min }+\Delta V_{s} f(t)\right]$

While the quantity $\Pi_{s}(t)$ behaves analogously to the solar wind speed, the solar wind density is inversely proportional to the solar wind speed.

The above described model neglects the fact, that the heliosphere is asymmetric with respect to an ecliptic-pole cut. The reason is that the solar wind is also asymmetric in the same aspect, i.e. the solar wind speed is fast over the poles, while it is slow in the ecliptic (e.g. in a cone with an opening angle $\Phi<35^{\circ}$ ). The mass flow is also conserved in this configuration, see e.g. McComas et al. (2000). In addition to that asymmetry, the solar cycle affects mainly the high speed component, which varies between $300 \mathrm{~km} / \mathrm{s}$ during solar maximum conditions to $800 \mathrm{~km} / \mathrm{s}$ during solar minimum conditions (McComas et al., 2004). 


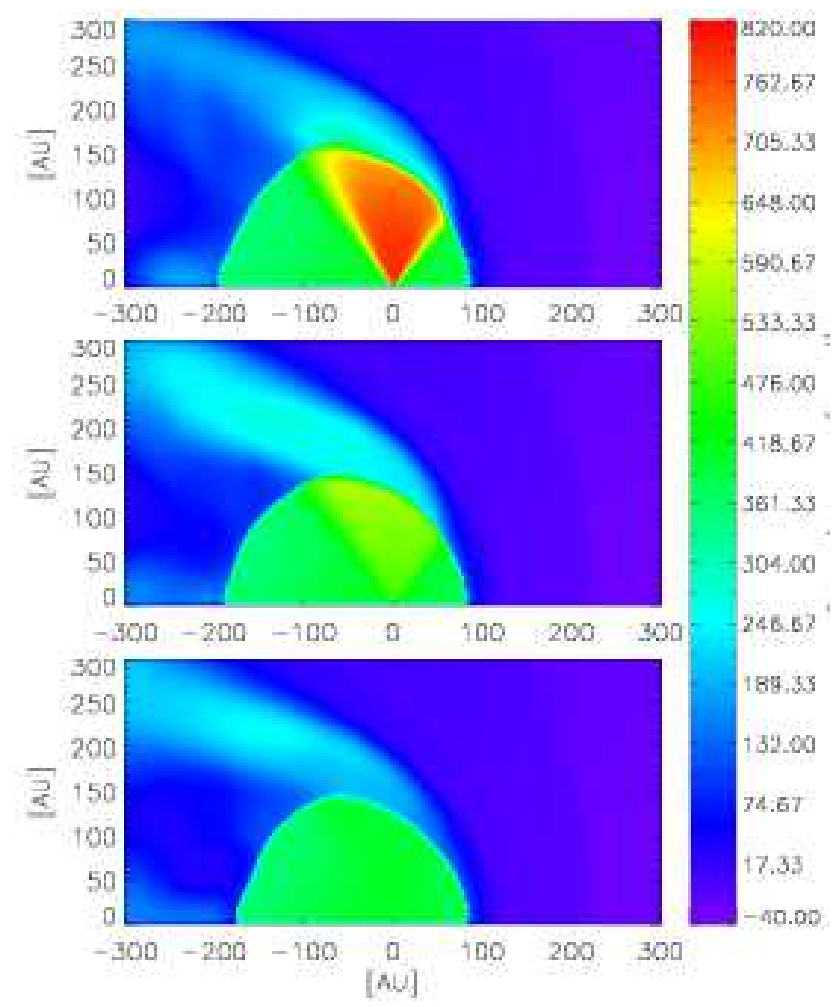

Fig. 1. Time evolution of the dynamic heliosphere represented by the solar wind speed in the meridional plane (Movie). In the upper panel the solar minimum condition is shown, e.g. fast solar wind over the poles. In the middle panel the rising phase and in the lower panel the solar maximum condition, e.g. slow solar wind from the ecliptic to the poles, are presented.

To simulate such an asymmetric behavior, we introduced the following function (Ferreira, 2002):

$$
\begin{aligned}
V_{s}= & V_{S, 0}(1 A U) \\
& \cdot\left[1-0.5 \cdot f(t) \cdot \tan h\left(\Delta_{c}\left[\theta-\theta_{c, 1}\right]\right)\right. \\
& \left.\quad+0.5 \cdot f(t) \cdot \tan h\left(\Delta_{c}\left[\theta+\theta_{c, 2}\right]\right)\right],
\end{aligned}
$$

where the constants are given here by: $\Delta_{c} R=8$, $\theta_{c, 1}=\theta_{c, 2}=35^{\circ}$ and $V_{s, 0}=400 \mathrm{~km} / \mathrm{s} . \quad \theta$ is the colatitude, e.g. $\theta=0$ is the pole, while $\theta=-\pi / 2$ gives the tail direction and $\theta=\pi / 2$ that of the nose.

In the following we will discuss the variations in the meridional plane, where the time dependence of the solar wind speed is given by its asymmetric form, Eq. (4) above. Three snapshots of the corresponding radial velocity and proton density profiles are shown in Fig. 1 and in Fig. 2. The complete time evolution can also be seen in a movie (please click here to see the movie).

\section{The Transport Model}

The modulation of ACR and GCR intensities in the dynamical heliospheric environment is modeled by a self-consistent

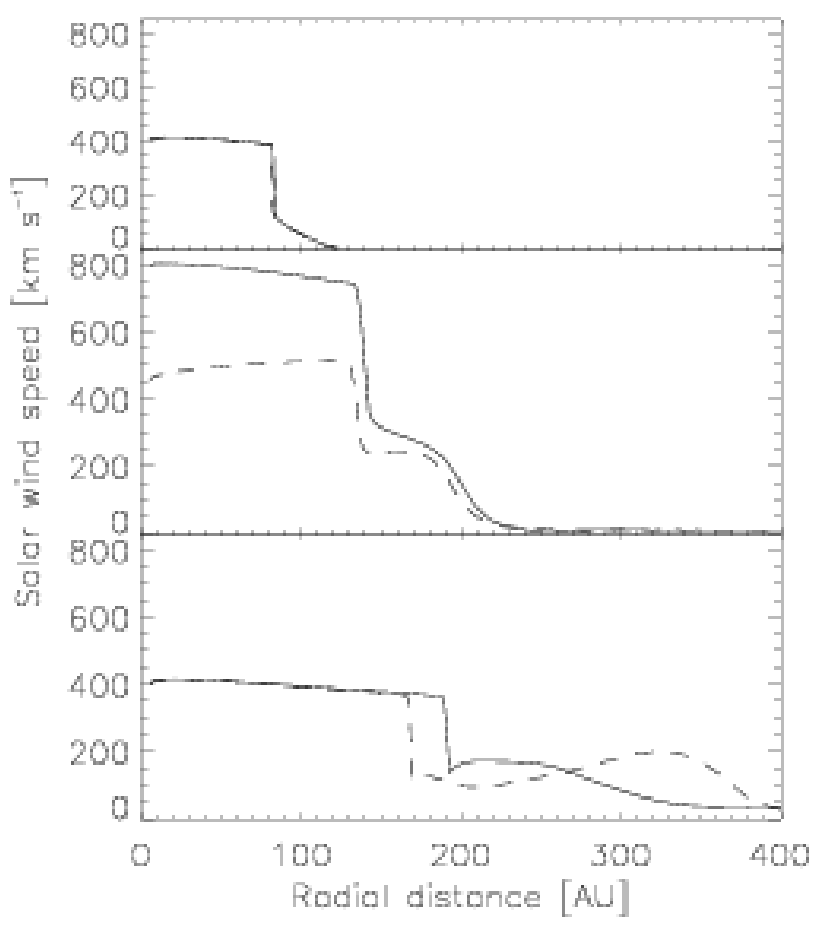

Fig. 2. Radial profiles of the solar wind speed for the equatorial regions in the heliospheric nose (top panel), the poles (middle panel) and the equatorial regions of the tail (bottom panel). The solid line corresponds to solar minimum conditions, and the dashed line to solar maximum conditions

hybrid model, where the cosmic ray intensities are calculated by solving time dependently the Parker (1965) transport equation:

$$
\begin{aligned}
\frac{\partial f}{\partial t}=-\left(\boldsymbol{V}-\left\langle\boldsymbol{v}_{D}\right\rangle\right) \cdot \nabla f & +\nabla \cdot\left(\overleftrightarrow{K}_{s} \cdot \nabla f\right) \\
& +\frac{1}{3}(\nabla \cdot \boldsymbol{V}) \frac{\partial f}{\partial \ln P}+Q
\end{aligned}
$$

in two spatial dimensions $(r, \theta)$ with $\theta$ the polar angle and $r$ the radial distance and $t$ time. Furthermore, $P$ is the rigidity, $Q$ any sources or sinks of cosmic rays inside the heliosphere, $\boldsymbol{V}$ the solar wind velocity, $\overleftrightarrow{K}_{s}$ the symmetric diffusion tensor and $f$ the omnidirectional distribution function with differential intensity $j=P^{2} f$ in units of particles $\mathrm{m}^{-2} \mathrm{~s}^{-1} \mathrm{sr}^{-1} \mathrm{MeV}^{-1}$. The pitch angle averaged guiding center drift velocity for a near isotropic cosmic ray distribution is given by $\left\langle\boldsymbol{v}_{D}\right\rangle=\nabla \times\left(\overleftrightarrow{K}_{A} \boldsymbol{e}_{B}\right)$ with $\boldsymbol{e}_{B}=\boldsymbol{B} / B_{m}$, where $\boldsymbol{B}$ is the Parker spiral (Parker, 1958) and $B_{m}$ is the magnitude of the modified background HMF (e.g. Jokipii and Kóta, 1989; Potgieter, 1999) and $\overleftrightarrow{K}_{A}$ is an antisymmetric tensor.

Equation (5) includes all the major transport processes: diffusion, convection, drifts and energy changes. Concerning the latter when $\nabla \cdot \boldsymbol{V}>0$ the particles are adiabatically cooled, which generally occurs in the upstream region of the solar wind termination shock. For $\nabla \cdot \boldsymbol{V}<0$, cosmic ray particles are accelerated via first order Fermi diffusive shock 


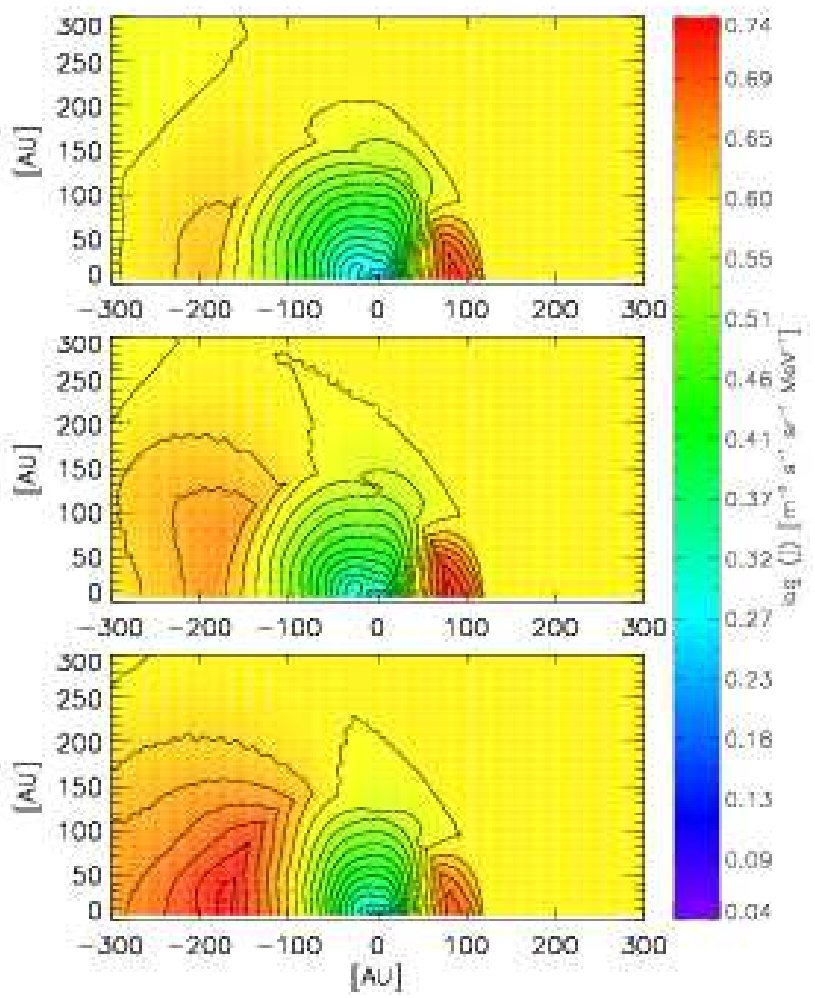

Fig. 3. Computed $1 \mathrm{GeV}$ GCR proton distribution for the $A<0$ polarity cycle when protons drift in from the equatorial plane toward the Sun. Shown from top to bottom are three different particle distributions corresponding to to the three different snapshots of heliosphere during various stages of solar activity as shown in Fig. (1)

acceleration at the termination shock, while for most of the downstream region in the heliosheath $\nabla \cdot \boldsymbol{V} \approx 0$ and almost no changes occurs.

The two most important transport processes in Eq. 5 which need to be highlighted here are cosmic ray diffusion and drifts. The corresponding coefficients of interest are found in the diffusion tensor $\boldsymbol{K}_{s}$ with the coefficients of special interest

$\kappa_{r r}=\kappa_{\|} \cos ^{2} \psi+\kappa_{\perp r} \sin ^{2} \psi$

$\kappa_{\theta \theta}=\kappa_{\perp \theta}$

$\kappa_{A}=\frac{\beta P}{3 B}$,

giving from top to bottom the radial and polar diffusion and drifts respectively, with the heliospheric magnetic field $B$ and the spiral angle $\psi$. Here $\kappa_{\|}$is diffusion parallel to the heliospheric magnetic field, $\kappa_{\perp r}$ perpendicular diffusion in the radial direction and $\kappa_{\perp \theta}$ perpendicular diffusion in the polar direction. Note that the heliospheric magnetic field is described by the Parker spiral field:

$B(t)=B_{0}(t) \frac{r_{0}^{2}}{r^{2}}\left(1,0, \frac{\Omega\left(r-r_{\odot}\right)}{V(t)} \sin \theta\right)$,

where $\Omega$ is the angular speed of the Sun, $r_{\odot}$ the solar radius, and $V(t)$ the time dependent solar wind speed described in

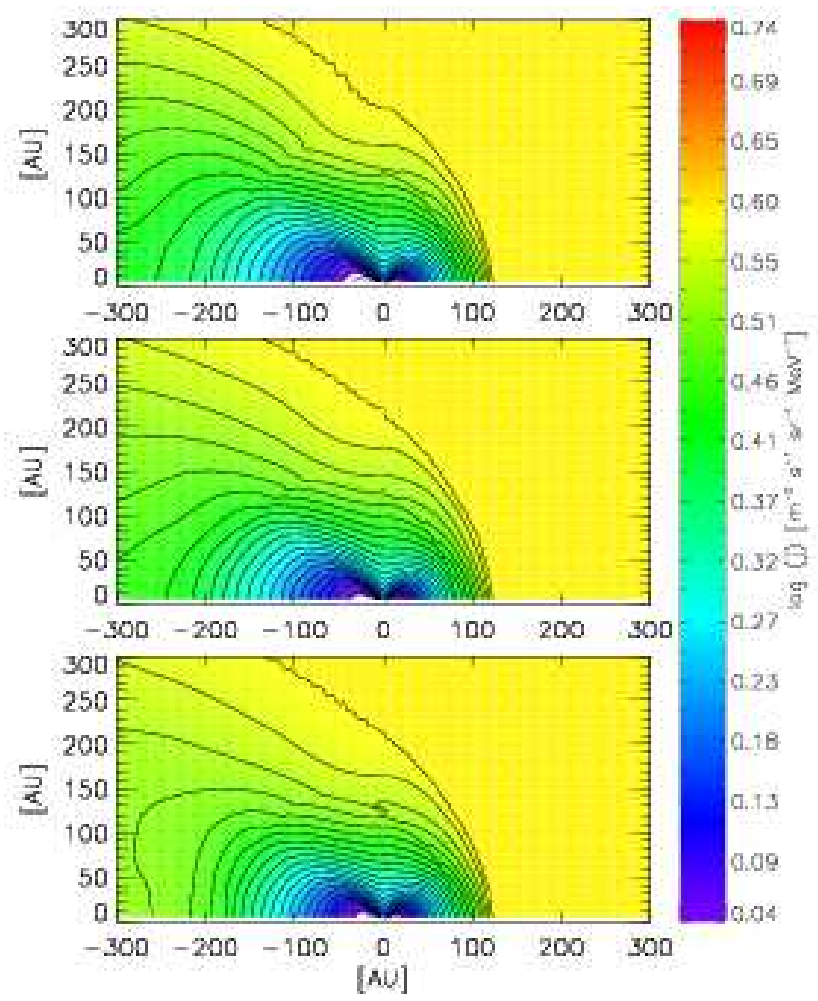

Fig. 4. Same as in Fig. (3) but for the $A>0$ polarity cycle

Eqs. (4) and (1). The spiral angle $\psi$ is then given by

$\tan \psi=\frac{\Omega\left(r-r_{\odot}\right)}{V(t)} \sin \theta$

Therefore any temporal changes in this quantity, due to changes in $V$, also has an effect on the radial diffusive transport and drifts processes because of the dependence on the spiral angle.

To calculate the cosmic ray spectra in the heliosphere, Eq. 5 is solved numerically via the Alternating Direction Implicit method (e.g. Douglas, 1962) whereafter the cosmic ray pressures are determined and taken into account when the hydrodynamic part is solved, as discussed above. For a detailed discussion on the cosmic ray transport parameters, see Ferreira et al. (2001) and Ferreira and Potgieter (2004) and Ferreira and Scherer (2004). However, it must be mentioned here that the effects of the heliospheric current sheet on cosmic ray transport are simulated as described in detail by Hattingh and Burger (1995). It was shown by Ferreira et al. (1999) that there exist no qualitative differences and insignificant quantitative differences between this approach and a three-dimensional approach including an actual wavy heliospheric current sheet. In the next section we focus on the dynamic changes in the computed GCR and ACR distributions in the heliosphere when a time-dependent solar wind profile is simulated. 


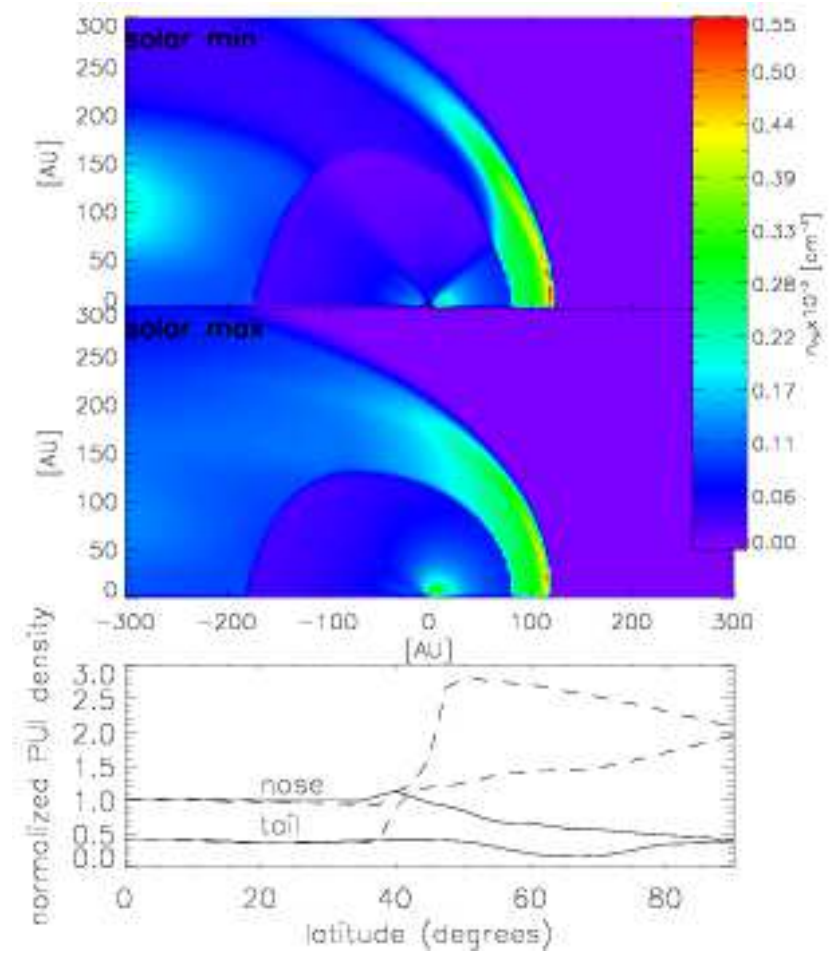

Fig. 5. Pickup Ion densities for both solar maximum (top panel) and solar minimum (middle panel) as computed by the hybrid model. The normalized densities at the termination shock, from which the ACR injection spectra are computed shown as a function of latitude in the bottom panel. The solid line corresponds to solar minimum, and the dashed line to solar maximum

\section{Model Results}

In the static case the GCR pressure calculated from the old 5-species Bonn model is almost identically to that calculated from the new hybrid model. The new feature for the GCRs is that now we are able to estimate the differential flux instead of an energy averaged flux. An example is discussed below.

The influence of the ACRs have been also modeled using an energy averaged flux, where the efficiency was described by a factor describing the fraction of PUIs converted into energetic particles. Now in the new hybrid code, we are able to calculate the acceleration from the PUIs to the ACRs in a self-consistent way. This changes the ACR pressure compared to that in the old model. The influence of the heliospheric structure is not negligible, but a detailed description of these processes would go far beyond this paper. The acceleration of ACRs and its influence to the structure of the heliosphere during both polarity cycles will be described in a separate paper. Here we will only show an example.

The main intention of this paper is to demonstrate that the hybrid code is working and will give new interesting inside in the physics of the heliosphere.

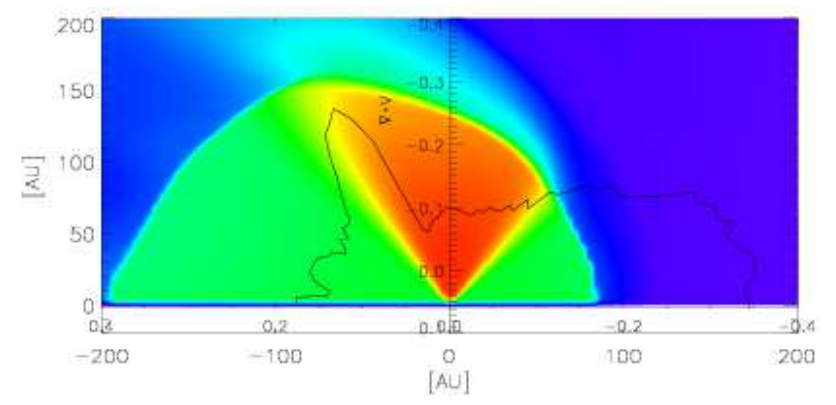

Fig. 6. The computed solar wind speed profile for solar minimum conditions as in the top panel of Fig. (1). Shown on top is the divergence of the solar wind speed at the termination shock radius, plotted as a polar plot.

\subsection{Dynamic Solar Wind}

Here we present the computations from the hybrid model with an asymmetric solar wind profile given by Eq. 4 showing the effects of solar cycle related changes on the geometry of the heliosphere, in particular the termination shock.

These are illustrated in Fig. (1), which shows the time evolution of the dynamic heliosphere with solar cycle related changes in the latitudinal profile of the solar wind. Shown here is the solar wind speed for selected periods over a 11-year cycle as three plots representing increasing solar activity from top to bottom. An interesting aspect is the so called "tornado alley" evident at high latitudes beyond the termination shock. In this narrow region the plasma speed significantly differs compared to that of the surrounding heliosheath plasma. During solar minimum, high speed wind flows into the tail regions of the outer heliosphere through this tornado alley. However, as the fast solar wind (solar minimum) over the poles disappears and only a uniformly slow solar wind (solar maximum) is left, this structure is less evident and almost disappears for extreme solar minimum periods (shown in the Movie). The effects of these dynamical solar cycle related changes on the solar wind density are illustrated quantitatively in Fig. (2) showing the radial profile of the solar wind speed for different polar angles. Shown at the top is computed values in the equatorial plane toward the heliospheric nose, middle panel shows values at the poles, and bottom panel shows values in the equatorial regions of the heliospheric tail. The solid line corresponds to solar minimum results, as in the top panel of Fig. (1), while the dashed line corresponds to values for solar maximum periods, corresponding to the bottom panel of Fig. (1). The most important feature shown here is that as solar activity increases the termination shock moves inward, especially at the polar and tail regions. This is because of the disappearance of the fast solar wind over the poles, and as shown below, this can have consequences on cosmic ray particle acceleration and distribution in these regions.

In this paper we can not give a detailed analysis of the tornado alley. It is in principle a similar feature as that describe 


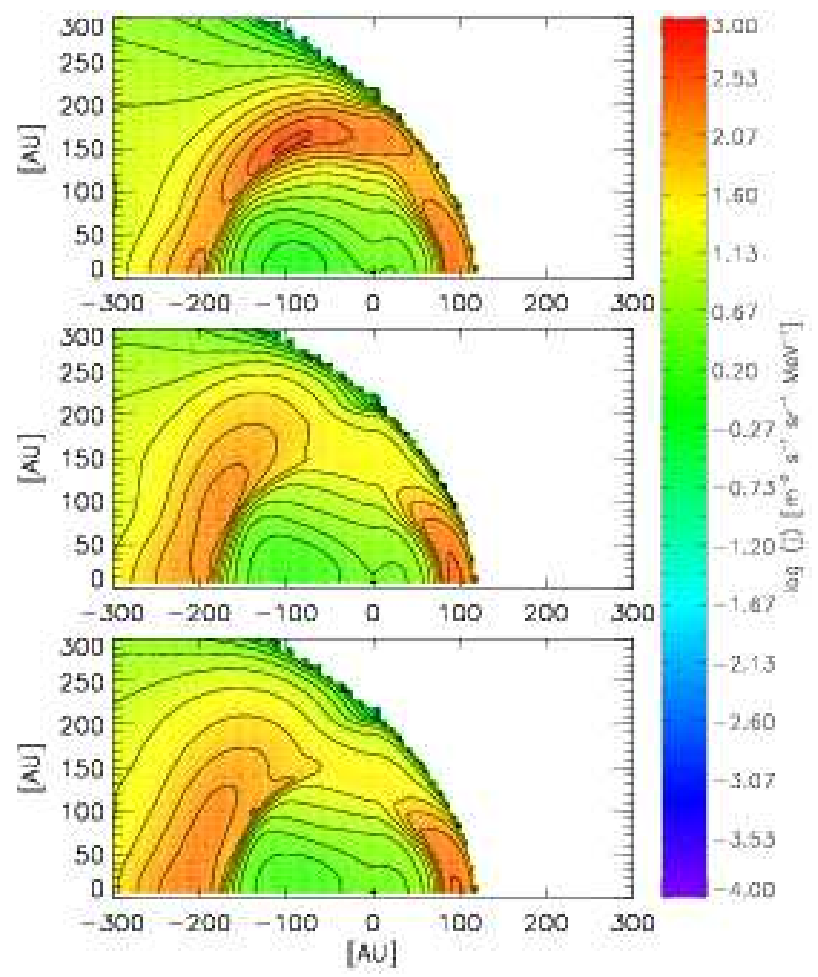

Fig. 7. Computed $30 \mathrm{MeV}$ ACR proton distribution for the $A<0$ polarity cycle. Shown from top to bottom are three different particle distributions corresponding to to the three different snapshots of heliosphere during various stages of solar activity as shown in Fig. (1)

Scherer and Fahr (in 2003a) in the tail region. While in the latter case the authors used Eq. (1) in the equatorial plane to describe the solar cycle variations, and found a complicated structure in the tail. In our case using an asymmetric solar wind speed in the meridional plane, this feature is restricted to the polar region, e.g. the tornado alley. The interaction with the solar wind hydrogen as well as the complicated shock structure at the beginning of the tornado alley and its time dependent behavior needs a much deeper description as can be given here. We will discuss it in detail in forthcoming publication.

\subsection{Galactic proton distribution}

Here we present computed GCR proton distributions corresponding to the three different snapshots of heliosphere during various stages of solar activity as shown in Fig. (1). For these computations the local interstellar proton spectrum (LIS) from Moskalenko et al. (2002), see also Langner (2004) is used. Figure (3) shows corresponding computed $1 \mathrm{GeV}$ GCR proton distributions for the $A<0$ polarity cycle, when protons drift in from the equatorial plane toward the Sun (click here to see the movie). Shown in the panels (from top to bottom) are particle distributions during minimum, moderate, and maximum solar conditions as shown in Fig. (1). Note that for this work only the dynamic changes

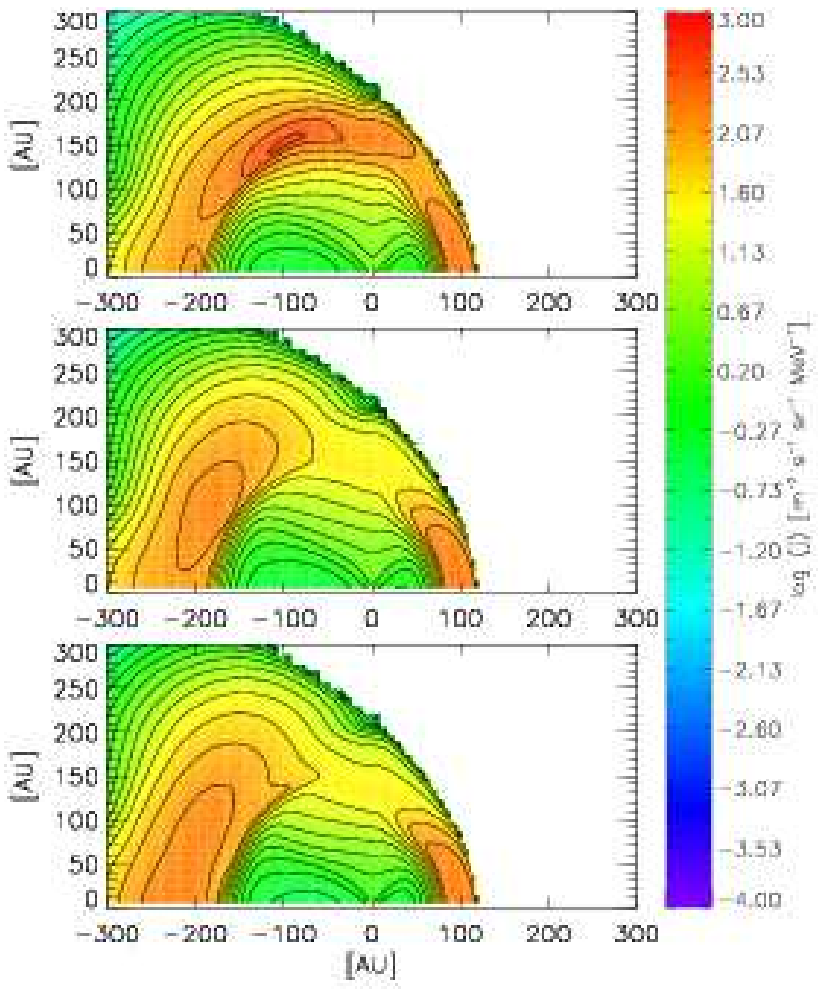

Fig. 8. Same as in Fig. (7) but for the $A>0$ polarity cycle

resulting from solar cycle related changes in the solar wind are taken into account. For future work a more realistic approach including corresponding changes in heliospheric current sheet tilt angle and diffusion coefficients over a solar cycle will be investigated.

Shown in Fig. (1) is that when solar activity increases, with the fast solar wind disappearing over the solar poles, the termination shock moves inward especially in the heliospheric pole and tail regions. This has a significant effect on the GCR particle distribution, as shown in Fig. (3). As the shock moves inward, more particles are accelerated with larger computed intensities in the equatorial regions of the heliospheric tail. For these solar maximum conditions there is a reduction in the nose tail asymmetry of the computed cosmic ray intensities due to the shock becoming more spherical compared to solar minimum. In fact the intensities at the shock in the heliospheric tail is now almost the same as in the nose regions. For the equatorial regions in the heliospheric nose there is almost no change in the cosmic ray distribution because in this region the termination shock radius is not sensitive to changes over the poles as shown in Fig. (1). It must again be emphasized that solar cycle related changes in the tilt angle and diffusion transport coefficients will alter model computations presented here, and this is only an illustration showing the effect on particle distribution of changes in the solar wind speed only.

Figure (4) shows the same as Fig. (3) except here computations are shown for the $A>0$ polarity cycle. For this cycle GCR protons drift in from the poles to the Sun and out- 
ward along the current sheet and as expected the effect of the termination shock on the computed intensities is much less evident due to the different drift direction. For this cycle, not so many protons are accelerated as they enter the heliosphere via the polar regions due to a weaker shock present, compared to the equatorial regions of the nose. Also shown here is that the dynamics of the solar wind speed mostly influence particle distribution in the heliospheric tail beyond the shock, with the computed intensities in this polarity cycle more dependent on plasma speed variations compared to results shown in Fig. (3).

\subsection{Anomalous proton distribution}

To compute ACR's a mono-energetic source was specified at the termination shock (see e.g. Steenberg and Moraal, 1996, 1999). As reported by Langner (2004) the corresponding solutions are independent of the injection energy as long as it is lower then the termination shock cutoff energy. Unique to the hybrid model is that the injection spectra are scaled to the computed pickup ion density which differs between the nose, pole and tail regions of the heliosphere as discussed above. This is shown in Fig. (5) which shows pickup ion densities for both solar maximum (top panel) and solar minimum (middle panel) as computed by the hybrid model. The normalized densities at the termination shock, from which the ACR injection spectra are computed, is shown as a function of latitude in the bottom panel. The solid line corresponds to solar minimum, and the dashed line to solar maximum.

These aspects influence the distribution of ACR's significantly over a solar cycle as shown in Fig. (7) (click here to see the movie). Presented here are the computed ACR distribution for the three different heliospheric geometries due to variations in the solar wind speed, shown in Fig. (1). It can be seen here that for this magnetic polarity, and for solar minimum conditions, the ACR's are accelerated at the termination shock in the equatorial regions, especially in the heliospheric nose. Interesting is that for solar minimum, intensity enhancements in ACR's also occur in the polar regions of the heliospheric tail. This is due to the fact that the value of the divergence of the polar solar wind speed approaches that in the equatorial region of the heliospheric nose. This interesting region is just below the "tornado alley" evident in the polar region and is shown in Fig. (6), which shows the computed solar wind speed profile for solar minimum conditions, as in the top panel of Fig. (1). Shown on top of the solar wind speed is its divergence at the termination shock as a polar plot. This large negative divergence in this region disappears toward solar maximum with the subsequent disappearance of the fast solar wind. The effect on the acceleration of ACR's is evident with the acceleration in this region disappearing for increasing solar activity.

As in the case for the GCR's the distribution in the heliospheric nose is not so much influenced by changing only the solar wind speed as a time dependent parameter. Also interesting is the effect of perpendicular diffusion in the polar region $\kappa_{\perp \theta}$ which contribute to changing particle inten- sities due to changes in the solar wind speed as shown by Ferreira et al. (2003). Because both the parallel $\kappa_{\| \mid}$and perpendicular diffusion coefficient in the radial direction $\kappa_{\perp r}$ depend on the spiral angle of the heliospheric magnetic field, which changes for different solar wind speeds, the net effect is that for solar maximum these are slightly smaller compared with solar minimum with the effect that the ratio of $\kappa_{\perp \theta} / \kappa_{\perp r}$ is now larger, resulting in changes in drift and latitudinal transport. These effects, together with the effect of changes in the divergence of the solar wind speed over a solar cycle are currently studied in more detail. Figure (8) shows the same as Fig. (7) except here computations are shown for the $A>0$ polarity cycle. Note that at these low energies $(30 \mathrm{MeV})$, model solutions are not as sensitive to drifts, as e.g. at $1 \mathrm{GeV}$ as shown in Figs. (1) and (2). As for the GCR computations, the ACR's differential fluxes in this polarity cycle are also largely reduced throughout the heliosphere due to less efficient acceleration at the termination shock. However, the acceleration in the polar regions of the heliospheric tail is still evident as for the $A<0$ polarity cycle. Also for increasing solar activity this disappears due to the disappearance of the fast solar wind. As for the $A<0$ polarity cycle the computed particle distribution inside the termination shock is mostly unaffected by dynamical changes in the solar wind speed, and the geometry of the termination shock.

\section{Conclusion and outlook}

In the present paper the first results of a hybrid model of the heliosphere is presented. It combines the hydrodynamic code for plasma and low energetic particles (e.g. hydrogen and PUIs) with the kinetic transport equation solving the transport and acceleration of high energy particles, e.g. GCRs and ACR's.

For the first time we could combine such models and, furthermore, study their dynamical behavior. We have shown how solar cycle related changes in the solar wind speed influences the geometry of the heliosphere and cosmic ray particle distribution within. However, in this paper we could only present the basic ideas and first results, but in forthcoming papers we will analyze the dynamics of the hybrid code, the Bonn-Potchefstroom (BoPo) model, further. Especially, the time dependent modulation of cosmic rays in such a realistic heliospheric environment will be studied.

Acknowledgement. We whish the thank Harm Moraal, Marius Potgieter, Hans Fahr and Horst Fichtner for valuable discussions. The authors are also grateful for financial support granted to them by the Deutsche Forschungsgemeinschaft in the frame of the project "Heliotrigger" (Fa 97/28-1) and from the South African National Research Foundation under grant number 2053475. This manuscript is also initiated as part of the forthcoming ISSI workshop on "The dynamic heliosphere, variable cosmic environments and their imprints in Earth's archives".

Edited by: T. Laitinen

Reviewed by: H.-R. Müller and S. Grzedzielski 


\section{References}

Alexashov, D. B., Chalov, S. V., Myasnikov, A. V., Izmodenov, V. V., and Kallenbach, R.: The dynamical role of anomalous cosmic rays in the outer heliosphere, Astron. Astrophys., 420, 729-736, 2004.

Axford, W. I.: Anisotropic diffusion of solar cosmic rays, Planet. Space Sci., 13, 1301-1309, 1965.

Axford, W. I., Leer, E., and Skadron, G.: The acceleration of cosmic rays by shock waves, Proc. 15th Int. Cosm. Ray Conf., Plovdiv, 11, 132-137, 1978.

Baranov, V. B. and Malama, Y. G.: Model of the solar wind interaction with the local interstellar medium - Numerical solution of self-consistent problem, J. Geophys. Res., 98, 15 157-15 163, 1993.

Baranov, V. B. and Zaitsev, N. A.: On the problem of the heliospheric interface response to the cycles of the solar activity, Geophys. Res. Lett., 25, 4051-4054, 1998.

Baranov, V. V., Krasnobaev, V., and Kulikovsky, A: A model of the interaction of the solar wind with the interstellar medium, Sov. Phys. Dokl., 15, 791-793, 1971.

Bell, A. R.: The Non-Linear Self-Regulation of Cosmic-Ray Acceleration at Shocks , Monthly Notices Roy. Astron. Soc., 225, 615-626, 1987.

Bieber, J. W., Matthaeus, W. H., Smith, C. W., Wanner, W., Kallenrode, M., and Wibberenz, G.: Proton and electron mean free paths: The Palmer consensus revisited, Astrophys. J., 420, 294 306, 1994.

Bieber, J. W., Matthaeus, W. H., Shalchi, A., and Qin, G.: Nonlinear guiding center theory of perpendicular diffusion: General properties and comparison with observation, Geophys. Res. Let., 31, 10 805, DOI: 10.1029/2004GL020077, 2004.

Blandford, R. D. and Ostriker, J. P.: Particle Acceleration by Astrophysical Shocks, Astrophys. J., 221, L29-L32, 1978.

Blum, P. W. and Fahr, H.-J.: Interaction between Interstellar Hydrogen and the Solar Wind, Astron. Astrophys., 4, 280-290, 1970.

Borrmann, T. and Fichtner, H.: On the dynamics of the heliosphere on intermediate and long time scales, Adv. Space Res. in press

Burger, R. A. and Hattingh, M.: Toward a Realistic Diffusion Tensor for Galactic Cosmic Rays, Astrophys. J., 505, 244-251, 1998.

Chalov, S. V. and Fahr, H.-J.: A two-fluid model of the solar wind termination shock modified by shock-generated cosmic rays including energy losses, Astron. Astrophys., 288, 973-980, 1994.

Cummings, A. C., Stone, E. C., and Steenberg, C. D.: Composition of Anomalous Cosmic Rays and Other Heliospheric Ions, Astrophys. J., 578, 194-210, 2002.

Donohue, D. J. and Zank, G. P.: Steady State and Dynamical Structure of a Cosmic-Ray- Modified Termination Shock, J. Geophys. Res., 98, 19005-19025, 1993.

Douglas, J.: Alternating direction implicit methods for three space variables, Numerische Mathematik, 4, 41-47, 1962.

Fahr, H.-J.: Neutral corpuscular energy flux by charge-transfer collisions in the vicinity of the sun, Astrophys. Spa. Sci., 2, 496503, 1968.

Fahr, H.-J.: Theory of interstellar medium diagnostics, Solar Wind Conference, 541-551, 1983.

Fahr, H.-J.: The Interstellar Gas Flow Through the Heliospheric Interface Region, Space Sci. Rev., 78, 199-212, 1996.

Fahr, H.-J.: The Multifluid Character of the 'Baranov' Interface, Astrophys. Space Sci., 274, 35-54, 2000.

Fahr, H.-J.: Global structure of the heliosphere and interaction with the local interstellar medium: three decades of growing knowledge, Adv. Space Res., 34, 3-13, 2004.

Fahr, H.-J., Nass, H. U., and Ruciński, D.: Drag-like effects of heliospheric neutrals due to elastic collisions, Astron. Astrophys., 142, 476-486, 1985.

Fahr, H.-J.: Local interstellar oxygen in the heliosphere - Its analytic representation and observational consequences, Astron. Astrophys., 241, 251-259, 1991.

Fahr, H.-J. and Ruciński, D.: Neutral interstellar gas atoms reducing the solar wind Mach number and fractionally neutralizing the solar wind, Astron. Astrophys., 350, 1071-1078, 1999.

Fahr, H.-J., Kausch, T., and Scherer, H.: A 5-fluid hydrodynamic approach to model the solar system-interstellar medium interaction, Astron. Astrophys., 357, 268-282, 2000.

Ferreira, S. E. S.: The heliospheric transport of galactic cosmic rays and Jovian electrons, PhD Thesis, Potchefstroom, South Afrika, 2002.

Ferreira, S. E. S. and Potgieter, M. S.: Long-term cosmic-ray modulation in the heliosphere, Astrophys. J., 603, 744-752, 2004.

Ferreira, S. E. S. and Scherer, K.: Modulation of Cosmic-Ray Electrons in the Outer Heliosphere, Astrophys. J., 616, 1215-1223, 2004b.

Ferreira, S. E. S., Potgieter, M. S., and Burger, R. A.: Comparison of a two- and three-dimensional drift model, Proc. 26th ICRC, 7, 77-80, 1999.

Ferreira, S. E. S., Potgieter, M. S., Burger, R. A., Heber, B., and Fichtner, H.: Modulation of Jovian and galactic electrons in the heliosphere. 1. Latitudinal transport of a few MeV electrons, J. Geophys. Res., 106, 24 979-24 988, 2001.

Ferreira, S. E. S., Potgieter, M. S., Moeketsi, D. M., Heber, B., and Fichtner, H.: Solar wind effects on the transport of $3-10 \mathrm{MeV}$ cosmic-ray electrons from solar minimum to solar maximum, Astrophys. J., 594, 552-560, 2003.

Ferreira, S. E. S., Potgieter, M. S., and Scherer, K.: Modulation of Cosmic-Ray Electrons in a Nonspherical and Irregular Heliosphere, Astrophys. J., 607, 1014-1023, 2004.

Fichtner, H.: Anomalous Cosmic Rays: Messengers from the Outer Heliosphere, Space Sci. Rev., 95, 639-754, 2001.

Fichtner, H.: Cosmic Rays in the Heliosphere: Progress in the Modeling During the Past Ten Years, Adv. Space. Res, in press.

Fisk, L. A., Kozlovsky, B. and Ramaty, R.: An Interpretation of the Observed Oxygen and Nitrogen Enhancements in Low-Energy Cosmic Rays, Astrophys. J., 190, L35-L37, 1974.

Florinski, V., Zank, G. P., and Pogorelov, N. V.: Galactic cosmic ray transport in the global heliosphere, J. Geophys. Res., 108, 1228 , DOI: 10.1029/2002JA009695, 2003.

Garcia-Munoz, M., Mason, G. M., and Simpson, J. A.: A New Test for Solar Modulation Theory: the 1972 May-July Low-Energy Galactic Cosmic-Ray Proton and Helium Spectra., Astrophys. J., 182, L81-L84, 1973.

Gazis, P. R.: Pioneer and Voyager observations of solar cycle variations in the outer heliosphere, Geophys. Res. Lett., 21, 17431746, 1994.

Giacalone, J. and Jokipii, J. R.: The Transport of Cosmic Rays Across a Turbulent Magnetic Field, Astrophys. J., 520, 204-214, 1999.

Hattingh, M. and Burger, R. A.: A new simulated wavy neutral sheet drift model, Adv. Space Res., 16, 213-216, 1995.

Holzer, T. E.: Neutral hydrogen in interplanetary space, Rev. Geophys. Space Phys., 15, 467-490, 1977.

Isenberg, P. A., Chih, P. P., and Fisk, L. A.:The heating of the solar wind by the interstellar neutral gas, J. Geophys. Res., 90, 12 040- 
$12046,1985$.

Izmodenov, V.: Velocity Distribution of Interstellar H Atoms in the Heliospheric Interface, Space Sci. Rev., 97, 385-388, 2001.

Izmodenov, V. V. and Malama, V.: Variations of interstellar H atom parameters in the outer heliosphere: solar cycle effects, Adv. Space Res., 34, 74-78, 2004.

Izmodenov, V. V., Geiss, J., Lallement, R., Gloeckler, G., Baranov, V. B., and Malama, Y. G.: Filtration of interstellar hydrogen in the two-shock heliospheric interface: Inferences on the local interstellar cloud electron density, J. Geophys. Res., 104, 4731-4742, 1999.

Jokipii, J. R.: Cosmic-Ray Propagation. I. Charged Particles in a Random Magnetic Field, Astrophys. J., 146, 480-487, 1966.

Jokipii, J. R. and Kota, J.: The polar heliospheric magnetic field, Geophys. Res. Let., 16, 1-4, 1989.

Kausch, T.: Ein hydrodynamisches Mehrkomponentenmodell zur Beschreibung der Wechselwirkung zwischen Heliosphäre und interstellarem Medium, Ph.D. thesis, Univ. of Bonn, Germany, 1998.

Karmesin, S. R., Liewer, P. C., and Brackbill, J. U.: Twodimensional simulations of the motion of the termination shock in response to 11 years variation in the solar wind, Geophys. Res. Let., 22, 1153-1156, 1995.

Kayser, S. E., Barnes, A., and Mihalov, D. J.: The far reaches of the solar wind - Pioneer 10 and Pioneer 11 plasma results, Astrophys. J., 285, 339-346, 1984.

Kissmann, R., Fichtner, H., Heber, B., Ferreira, S. E. S., and Potgieter, M. S.: First results of a new 3-D model of the timedependent modulation of electrons in the heliosphere, Adv. Space Res., 32, 681-686, 2003.

Kòta, J. and Jokipii, J. R.: Modeling of 3-D Corotating CosmicRay Structures in the Heliosphere, Space Sci. Rev., 83, 137-145, 1998.

Krimigis, S. M., Decker, R. B., Hill, M. E., Armstrong, T. P., Gloeckler, G., Hamilton, D. C., Lanzreotti, L. J., and Roelof, E. C.: Voyager 1 exited the solar wind at a distance of 85 AU from the Sun, Nature, 426, 45-48, 2003.

Lazarus, A. and Belcher, J.: Large-Scale Structure of the Distant Solar Wind and Heliosphere, Solar Wind 6, Colorado/USA, edited by Pizo, Holzer, and Scime, 533-546, 1987.

Lazarus, A. J. and McNutt, R. L.: Plasma observations in the distant heliosphere - A view from Voyager, in "The Outer Heliosphere", COSPAR Colloquium No.1, edited by Grzedzielski, S. and Page, E., Warsaw, Poland, 229-240, 1990.

Langner, U. W.: Effects of termination shock acceleration on cosmic rays in the heliosphere, $\mathrm{PhD}$ thesis, North-West University, South Africa, 2004.

Lerche, I. and Schlickeiser, R.: Cosmic ray transport in anisotropic magnetohydrodynamic turbulence. I. Fast magnetosonic waves, Astron. Astrophys., 378, 279-294, 2001.

le Roux, J. A., Zank, G. P., and Ptuskin, V. S.: An Evaluation of Perpendicular Diffusion Models Regarding Cosmic-Ray Modulation on the Basis of a Hydromagnetic Description of Solar Wind Turbulence, J. Geophys. Res., 104, 24 845-24 862, 1999.

Liewer, P. C., Rath, S., and Goldstein, B. E.: Hybrid simulations of interstellar pickup ion acceleration at the solar wind termination shock, J. Geophys. Res., 100, 19 809-19818, 1995.

Linde, T. J., Gombosi, T. I., Roe, P. L., Powell, K. G., and Dezeeuw, D. L.: Heliosphere in the magnetized local interstellar medium - Results of a three-dimensional MHD simulation, J. Geophys. Res., 103, 1889-1904, 1998.

McComas, D. J., Barraclough, B. L., Funsten, H. O., Gosling, J. T.,
Santiago-Muñoz, E., Skoug, R. M., Goldstein, B. E., Neugebauer, M., Riley, P., and Balogh, A.: Solar wind observations over Ulysses' first full polar orbit, J. Geophys. Res., 105, 10419 $10434,2000$.

McComas, D. J., Elliott, H. A., Schwadron, N. A., Gosling, J. T., Skoug, R. M., and Goldstein, B. E.: The three-dimensional solar wind around solar maximum, Geophys. Res. Lett., 30, 24-1, 2003.

McComas, D., Allegrini, F., Bochsler, P., Bzowski, M., Collier, M., Fahr, H., Fichtner, H., Frisch, P., Funsten, H., Fuselier, S., Gloeckler, G., Gruntman, M., Izmodenov, V., Knappenberger, P., Lee, M., Livi, S., Mitchell, D., Möbius, E., Moore, T., Reisenfeld, D., Roelof, E., Schwadron, N., Wieser, M., Witte, M., Wurz, P., and Zank, G.: The Interstellar Boundary Explorer (IBEX), AIP Conf. Proc. 719: Physics of the Outer Heliosphere, 162-181, 2004

McDonald, F. B., Stone, E. C., Cummings, A. C., Heikkila, B., Lal, N., and Webber, W. R.: Enhancements of energetic particles near the heliospheric termination shock, Nature, 426, 48-51, 2003.

Moskalenko, I. V., Strong, A. W., Ormes, J. F., and Potgieter, M. S.: Secondary antiprotons and propagation of cosmic rays in the galaxy and heliosphere, Astrophys. J., 565, 280-296, 2002.

Müller, H.-R., Zank, G. P., and Lipatov, A. S.: Self-consistent hybrid simulations of the interaction of the heliosphere with the local interstellar medium, J. Geosphys. Res., 105, 27 419-27 438, 2000.

Osterbart, R. and Fahr, H.-J.: A Boltzmann-kinetic approach to describe the entrance of neutral interstellar hydrogen into the heliosphere, Astron. Astrophys., 264, 260-269, 1992.

Parker, E. N.: Dynamics of the Interplanetary Gas and Magnetic Fields, Astrophys. J., 128, 664-676, 1958

Parker, E. N.: Interplanetary dynamical processes, NY, Interscience Publishers, 1963.

Parker, E. N.: The passage of energetic charged particles through interplanetary space, Planet. Space Sci., 13, 9-49, 1965.

Pauls, H. L. and Zank, G. P.: Interaction of a nonuniform solar wind with the local interstellar medium 2. A two-fluid model, J. Geophys. Res., 102, 19779-19788, 1997.

Pesses, M. E., Eichler, D., and Jokipii, J. R.: Cosmic-Ray Drift, Shock Wave Acceleration, and the Anomalous Component of Cosmic Rays, Astrophys. J., 246, L85-L88, 1981.

Phillips, J. L., Bame, S. J., Barnes, A., Barraclough, B. L., Feldman, W. C., Goldstein, B. E., Gosling, J. T., Hoogeveen, G. W., McComas, D. J., Neugebauer, M., and Suess, S. T.: Ulysses Solar Wind Plasma Observations from Pole to Pole, Geophys. Res. Lett., 22, 3301-3304 1995.

Pogorelov, N. V. and Matsuda, T.: Influence of the interstellar magnetic field direction on the shape of the global heliopause, J. Geophys. Res., 103, 237-245, 1998.

Pogorelov, N. V. and Matsuda, T.: Nonevolutionary MHD shocks in the solar wind and interstellar medium interaction, Astron. Astrophys., 354, 697-702, 2000.

Pogorelov, N. V., Zank, G. P., and Ogino, T.: Three-dimensional Features of the Outer Heliosphere Due to Coupling between the Interstellar and Interplanetary Magnetic Fields. I. Magnetohydrodynamic Model: Interstellar Perspective, Astrophys. J., 614, 1007-1021, 2004.

Potgieter, M. S.: Time-dependent cosmic-ray modulation - Role of drifts and interaction regions, Adv. Space Res., 13, 239-249, 1993.

Potgieter, M. S.: The long-term modulation of galactic cosmic rays 
in the heliosphere, Adv. Space Res., 16, 191-203, 1995.

Potgieter, M. S.: The Modulation of Galactic Cosmic Rays in the Heliosphere, Space Sci. Rev., 83, 147-158, 1998.

Potgieter, M. S.: The heliospheric modulation of cosmic rays protons: The role of enhanced perpendicular diffusion during periods of minimum solar modulation, Adv. Space Res., 23, 3, 449452, 1999.

Ratkiewicz, R., Barnes, A., Molvik, G. A., Spreiter, J. R., and Stahara, S. S.: Heliospheric termination shock motion due to fluctuations in the solar wind upstream conditions: Spherically symmetric model, J. Geophys. Res., 101, 27 483-27 498, 1996.

Ratkiewicz, R., Barnes, A., Molvik, G. A., Spreiter, J. R., Stahara, S. S., Vinokur, M., and Venkateswaran, S.: Effect of varying strength and orientation of local interstellar magnetic field on configuration of exterior heliosphere: 3D MHD simulations, Astron. Astrophys., 335, 363-369, 1998.

Ripken, H. W. and Fahr, H.-J.: Modification of the local interstellar gas properties in the heliospheric interface, Astron. Astrophys., 122, 181-192, 1983.

Ruciński, D. and Fahr, H.-J.: Nonthermal ions of interstellar origin at different solar wind conditions, Ann. Geophys., 9, 102-110, 1991.

Scherer, K. and Fahr, H.-J.: Breathing of heliospheric structures triggered by the solar-cycle activity, Ann. Geophys., 21, 13031313, 2003a, Sref-ID: 1432-0576/ag/2003-21-1303.

Scherer, K. and Fahr, H.-J.: Remote diagnostic of the solar-cycleinduced heliospheric interface variation using energetic neutral atoms, Astron. Astrophys. 404, L47-L50, 2003b.

Scherer, K. and Fichtner, H.: Constraints on the heliospheric magnetic field variation during the Maunder Minimum from cosmic ray modulation modeling, Aston. Astrophys., 413, L11-L14, 2004

Scherer, K., Fahr, H.-J., Fichtner, H., and Heber, B.: Long-term modulation of cosmic rays in the heliosphere and its influence at earth, Sol. Phys., in press

Steenberg, C. D.: Modeling of anomalous and galactic cosmic ray modulation in the heliosphere, $\mathrm{PhD}$ thesis, Potchefstroom University for Che, South Africa, 1998.
Steenberg, C. D. and Moraal, H.: An acceleration/modulation model for anomalous cosmic ray hydrogen in the heliosphere, Astrophys. J., 463, 776-783, 1996.

Steenberg, C. D. and Moraal, H.: Form of the anomalous cosmic ray spectrum at the solar wind termination shock, J. Geophys. Res., 104, 24 879-24 884, 1999.

Steinolfson, R. S.: Termination shock response to large-scale solar wind fluctuations, J. Geophys. Res., 99, 13 307-13 314, 1994.

Suess, S. T.: The heliopause, Rev. Geophys., 28, 97-115, 1990.

Tanaka, T. and Washimi, H.: Solar cycle dependence of the heliospheric shape deduced from a global MHD simulation of the interaction process between a nonuniform time-dependent solar wind and the local interstellar medium, J. Geophys. Res., 104, 12 605-12 616, 1999.

Wang, C. and Belcher, J. W.: The heliospheric boundary response to large-scale solar wind fluctuations: A gasdynamic model with pickup ions, J. Geophys. Res., 104, 549-556, 1999.

Washimi, H. and Tanaka, T.: 3-D Magnetic Field and Current System in the Heliosphere, Space Sci. Rev., 78, 85-94, 1996.

Whang, Y. C. and Burlaga, L. F.: Termination shock: solar cycle variations of location and speed, J. Geophys. Res., 98, $15221-$ $15230,1993$.

Wu, F. M. and Judge, D. L.: Temperature and flow velocity of the interplanetary gases along solar radii, Astrophys. J., 231, 594605, 1979.

Zank, G. P.: Interaction of the solar wind with the local interstellar medium: a theoretical perspective, Space Sci. Rev., 89, 413-688, 1999.

Zank, G. P. and Pauls, H. L.: Modelling the Heliosphere, Space Sci. Rev., 78, 95-106, 1996.

Zank, G. P. and Pauls, H. L.: Shock propagation in the outer heliosphere 1. Pickup ions and gasdynamics, J. Geophys. Res., 102, 7037-7050, 1997.

Zank, G. P. and Müller, H.-R.: The dynamical heliosphere, J. Geophys. Res., 108, SSH 7-1, doi: 10.1029/20002JA009689, 2003.

Zhang, M.: A Markov Stochastic Process Theory of Cosmic-Ray Modulation, Astrophys. J., 513, 409-420, 1999a.

Zhang, M.: A Path Integral Approach to the Theory of Heliospheric Cosmic-Ray Modulation, Astrophys. J., 510, 715-725, 1999 b. 